\title{
Calculation of the height parameters of the treated surface roughness under the influence of the flow of abrasive particles
}

\author{
Georgi Tzordanidi ${ }^{1}$, Oksana Pyatnitzkaya ${ }^{1}$, Elena Fisunova $^{1}$, Tatyana Lavrenova ${ }^{1, *}$ and \\ Olga Baryshnikova ${ }^{1}$ \\ ${ }^{1}$ FGBOU VO «Don State Technical University» (DSTU), 344000, Rostov-on-Don, Russian \\ Federation
}

\begin{abstract}
A method for calculating the roughness of the treated surface for various methods of processing parts flow of abrasive particles, taking into account the random nature of the formation of the treated surface relief.
\end{abstract}

The absence of a rigid connection between the particles in the finishing processing of parts by the flow of abrasive particles eliminates the possibility of presenting the formation of the treated surface as the final result of the interaction of two rough surfaces-the part and the tool, which is based on the successive multiple imposition of elementary cutting tool profiles with rigidly fixed abrasive grains, forming a conditional profile called "effective cutting profile" $[1,2,3,4]$. However, common to all varieties of finishing abrasive treatment is to reduce the height parameters of the initial roughness during the processing period to a value that characterizes the process of reproduction of the established roughness, in which further reduction of these parameters is impossible without changing the processing conditions. The analysis of profilograms of surfaces allows to claim that at the stage preceding formation of the established roughness, decrease in height of roughnesses (Fig. 1, a and b) is due to intensive removal of metal from their tops. If we assume that the volume of the removed metal is equal in magnitude to the volume of the vertices of the irregularities smoothed as a result of processing by the value $\lambda$ [6], the height of the irregularities of the treated surface $R_{\max }^{\text {treated }}$ can be calculated by the formula:

$$
R_{\text {max }}^{\text {treated }}=R_{\text {max }}^{\text {initial }}-\lambda
$$

The imposition of many traces of processing and their mutual overlap lead to the formation of a surface relief, the parameters of which do not depend on the direction of measurement. This makes it possible to describe the roughness of such a surface by a homogeneous normal and isotropic random field having an ergodic property. The latter means that any sufficiently long cross-section of the field by a plane normal to the median

\footnotetext{
* Corresponding author: bys ka87@mail.ru
} 
plane is a reliable implementation that allows to obtain all the characteristics of the random function from the profilogram of the corresponding length. According to the results of earlier studies, for the roughness described by a homogeneous normal random field with a continuous correlation function and a zero mean value, the value of the volume $W_{U}$, located above the level $U$, can be found by integrating the cross-sectional area of the surface $A(U)$ at all levels above the level $U$, counted

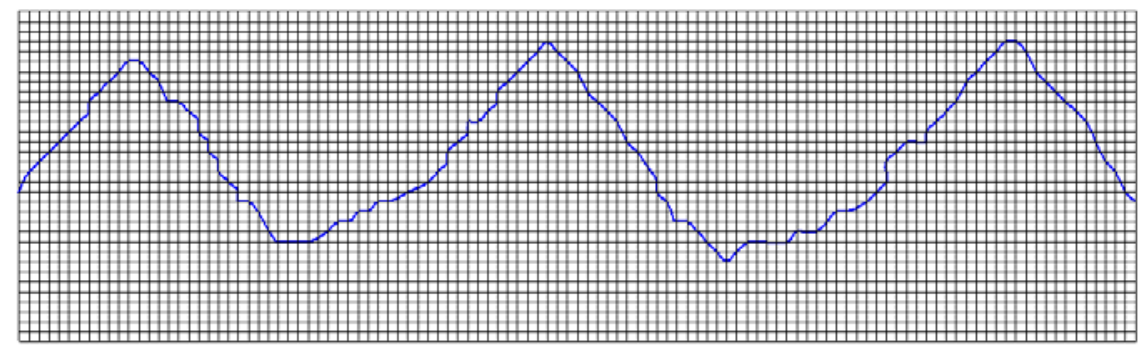

a)

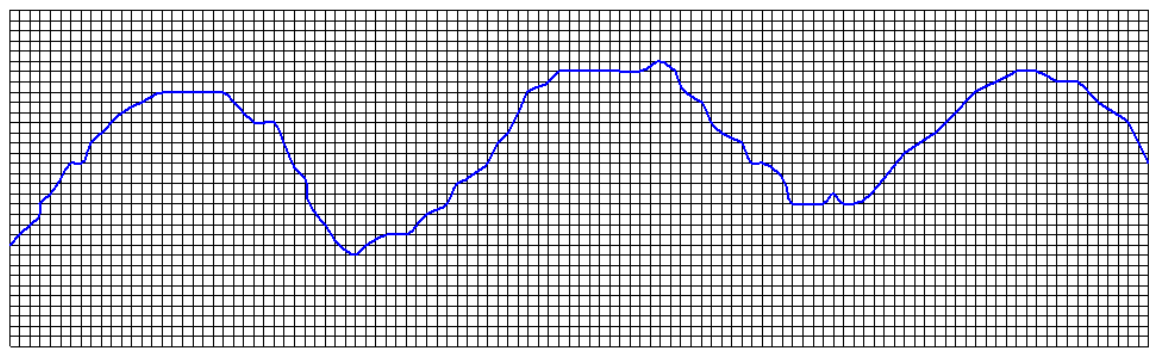

b)

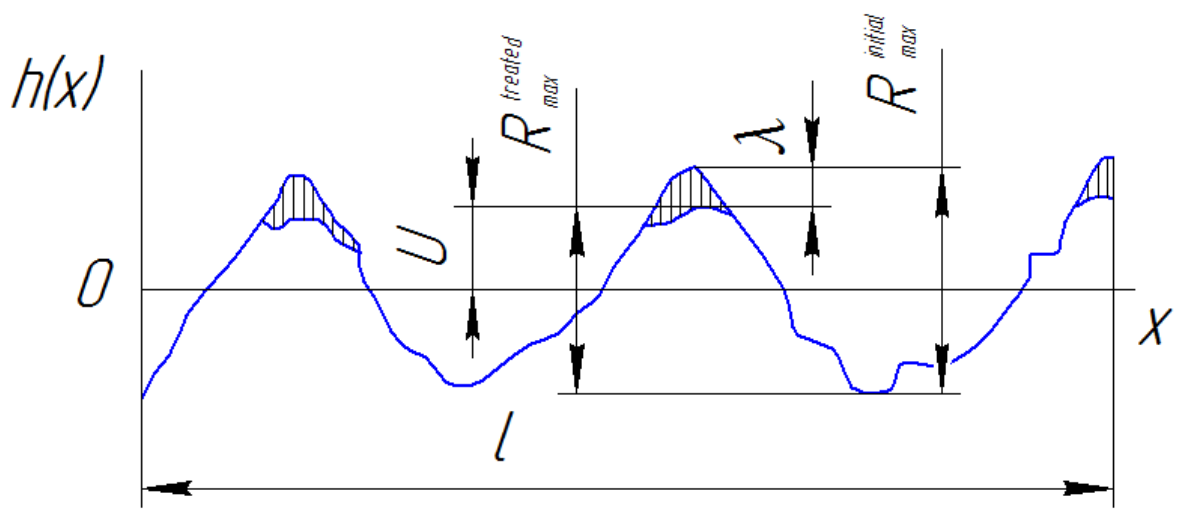

c)

Fig. 1. Model of roughness formation of the treated surface: a - profilogram original roughness; b-profilogram of the surface after treatment; b-calculation scheme.

the median plane (Fig.1, c)

$$
W_{U}=\int_{U}^{\infty} A(U) d U,
$$


and the expectation of this value

$$
\begin{gathered}
E\left\{W_{U}\right\}=A \int_{U}^{\infty}\left[1-\Phi\left(\frac{U}{\sigma}\right)\right] d U \\
=\sigma A\left\{\exp \left(-\frac{U^{2}}{2 \sigma^{2}}\right) \frac{1}{\sqrt{2 \pi}}-\frac{U}{\sigma}\left[1-\Phi\left(\frac{U}{\sigma}\right)\right]\right\},
\end{gathered}
$$

where $\sigma$ is the standard deviation of the profile;

$A$ - the area of the treated surface of the product.

$$
\Phi\left(\frac{U}{\sigma}\right)=\frac{1}{\sqrt{2 \pi}} \int_{-\infty}^{U / \sigma} \exp \left(-\frac{Z^{2}}{2}\right) d Z-\text { Laplace function. }
$$

Since there is a simple connection between $\lambda$ and $U$

$$
\lambda=\frac{1}{2} R_{\max }^{\text {initial }}-U,
$$

expression (1) will take the form

$$
R_{\max }^{\text {treated }}=\frac{1}{2} R_{\max }^{\text {initial }}+U
$$

To use the expression (4), it is necessary to find $U$ from the transcendental equation (3), which can be solved only approximately. Considering that $E\left\{W_{U}\right\}$ linear with respect to $\sigma$. $A$, but $R_{\max }=6 \sigma$, calculate the value of the function

$$
\xi(t)=\frac{1}{\sqrt{2 \pi}} \exp \left(-\frac{U^{2}}{\sigma^{2}}\right)-\frac{U}{\sigma}\left[1-\Phi\left(\frac{U}{\sigma}\right)\right]
$$

in the range $-2,5<\frac{U}{\sigma}=t<2,5$ and plot it. Strictly speaking, we should consider the behavior of this function in the interval $-3<\frac{U}{\sigma}<3$, however, this would mean no metal removal at $U / \sigma=3$ and a reduction in the initial roughness $R_{\text {max }}^{\text {initial }}$ до 0 при $\frac{U}{\sigma}=-3$, I. е. both outcomes are unlikely.

Graph of function $\xi(t)$ (Fig. 2) allows to predict the type of approximating dependence:

$$
\xi(t)=\alpha e^{\beta t}
$$

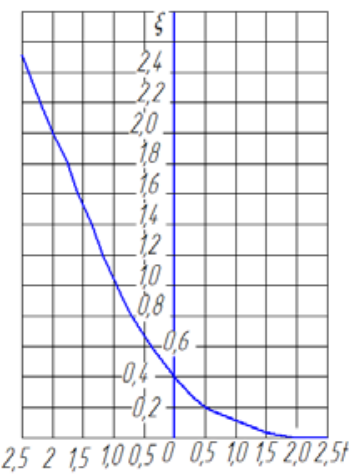

Fig. 2. Function Graph $\xi(t)$. 
The values of the coefficients $\alpha$ and $\beta$ are determined by the least squares method [5].

Note that the approximation by dependence (6) has low accuracy in the region of negative $U / \sigma$. Therefore, in order to improve the accuracy of further calculations, we divide the area of interest of the values of the function $\xi(t)$ into two intervals $-2,5<\frac{U}{\sigma}<0$ u $0<\frac{U}{\sigma}<2,5$. With their approximarely expressions:

$$
\left.\hat{\xi}(\mathrm{t})\right|_{2,5} ^{0}=0,093 \mathrm{t}^{2}+0,42,
$$

and

$$
\left.\hat{\xi}(\mathrm{t})\right|_{2,5} ^{0}=0,42 \mathrm{e}^{-1,71 \mathrm{t}} .
$$

The values of the function $\xi(t)$ calculated by the formulas (5), (7), (8) and the corresponding errors indicate that the greatest accuracy of the approximation is realized in the interval $-2,5<\frac{U}{\sigma}<1,5$, covering most of the area of values achievable as a result of processing $\frac{U}{\sigma}$.

Having the values of the volume of the removed metal and one of the parameters of the initial roughness $R_{\max }, \sigma$ or $R_{a}$, and considering $U=t \sigma$, it is easy to define $U$ by one of the expressions

$$
\frac{W_{U}}{\sigma A}=0,093 t^{2}-0,6 t+0,42
$$

or

$$
\frac{\mathrm{W}_{\mathrm{U}}}{\sigma \mathrm{A}}=0,42 \mathrm{e}^{-1,71 \mathrm{t}} .
$$

To decide which of them to use, you must first calculate the auxiliary value

$$
\widehat{U}=\frac{W}{A},
$$

representing the average height of the layer of the removed material. In this case, when $0<\frac{\widehat{U}}{R_{\text {max }}^{\text {initial }}} \leq 0,5$ the value $U$ should be calculated by the formula (10), if $0,5<\frac{\widehat{U}}{R_{\text {max }}^{\text {initil }}} \leq$ 1 , it is determined from (7).

Substituting $U$ in (2), we find $R_{\max }^{\text {treated }}$, corresponding to the period preceding the beginning of the reproduction of the established roughness $\mathrm{T}_{\text {stated }}$. Obviously, for a period of time $t>\mathrm{T}_{\text {stated }}$, calculated value $R_{\text {max }}^{\text {treated }}$ will be understated or even absurd, so you should always keep in mind that the minimum achievable value $\mathrm{R}_{\max }^{\text {treated }}$ cannot be less than the maximum depth of introduction of abrasive particles of the given granularity corresponding to the set modes of processing and defining size $R_{\max }$ of the established roughness, i.e., of the

$$
0,34 V_{H} \sqrt{\frac{m}{\rho \sigma \mathrm{T}}} \leq R_{\max }^{\text {treated }}
$$

The rest of the height parameters can be calculated using the dependencies that establish the relationship between $R_{\max }$ and $\sigma, R_{\mathrm{a}}$ and $\sigma, R_{\mathrm{a}}$ and $R_{z}$. Так $R_{\max }=6 \sigma ; R_{a}=\sqrt{\frac{2}{\pi}} \sigma$; $R_{z} \approx 5 R_{a}$. 


\section{Conclusions}

1. The formation model of treated surface roughness has been created described by a homogeneous normal random field with a method of parameter calculation $R_{\max }, R_{\mathrm{a}}$ $R_{z}$.

2. The possibility of productivity management for and surface quality by work condition changing and final spindle movement.

\section{References}

1. Lipsky G.K., Sheftel B.T. Researches of radial vibration for the bearing in forms in rolling surface by spectral analyses method.-Bearing production, (2016) ,№6, p.39.

2. Stratievsky I.H., Kremen Z.I. The calculation of metal put out during treatment by abrasive bars. Works of ISRIM№14.M.-L.Machinebuilding, (2013), p.7-14/

3. Filimonov L.N. About the role of circle work surface in the process of treatment. Book:

4. Possible-statistic bases of treatment processes and final stage: Common Institute list of papers.L.(1974), p125-128

5. Filimonov L.N. Effective cutting profil of treated circles and its role in treated part of upper layer formation,-Abrasives , (2015) , № 2, p.30-35/

6. Babichev A.P. Some questions of vibrating treatment theory for maching parts and tools.-in Book: Position and perspectives of productive investigation for treatment by vibration.Rostov n/D, (1974), p. 3-9.

7. Melentjeva P.V. Approximate Calculation.-M.:Physmathstpubl, (2010).-388p. 